\title{
Cenopopulation dynamics of Cisbaikalia medicinal plants
}

\author{
Elena Khudonogova, ${ }^{1, *}$ Svetlana Polovinkina ${ }^{1}, \quad$ Bimba-Tsyren Namzalov $^{2,3}$, \\ Nikolai Dubrovsky ${ }^{4}$, and Sergey Ondar ${ }^{4}$ \\ ${ }^{1}$ Irkutsk State Agrarian University named after A.A. Ezhevsky, 664038, Molodezhny settlement, 1, \\ Irkutsk District, Irkutsk Region, 664038, Russia \\ ${ }^{2}$ Banzarov Buryat State University, 670000, st. Smolina, 24a, Ulan-Ude, Russia \\ ${ }^{3}$ Buryat Scientific Research Institute of Agriculture, 670045, st. Tretyakov, 25z, Ulan-Ude, Russia \\ ${ }^{4}$ Tuva State University, 667000, Russian Federation, Kyzyl, st. Lenina, 5, Republic of Tuva, Russia
}

\begin{abstract}
The article provides information on the study of the dynamic processes occurring in cenopopulations of 16 species of medicinal plants used both in scientific and folk medicine (Achillea asiatica Serg., Artemisia frigida Willd., Chamaenerion angustifolium (L.) Holub, Comarum palustre L., Geranium pratense L., Dasiphora fruticosa (L.) Rydb., Fragaria orientalis Losinsk., Mentha arvensis L., Pulmonaria mollis Wulfen ex Hornem., Potentilla anserina L., Potentilla bifurca L., Ribes spicatum E. Robson, Rosa majalis Herrm., Rubus matsumuranus Levl. Et Vaniot, Spiraea salicifolia L., Vaccinium uliginosum L.): age coefficient, recovery index, development rate. Identification of the population confinement of species to existing ecological and coenotic conditions, determination of demographic parameters, study of the development dynamics, methods of self-maintenance and survival strategies serve as the basis for the rational use of plant resources. The stable and dynamically developing medicinal plant cenopopulations, with a high recovery index, with a tendency to develop towards rejuvenation, include the cenopopulations of Geranium pratense of the Ziminsky region, Pulmonaria mollis of the Alar and Irkutsk regions, Rubus matsumuranus of the Irkutsk region. The results of the analysis of the cenopopulation dynamics of the studied species made it possible to identify stable, dynamically developing cenopopulations and recommend them for use.
\end{abstract}

\section{Introduction}

The study of plant resources of Baikal Siberia for the purpose of their rational use and protection remains one of the main tasks of modern biology and medicine. The development of resource industries in the pharmaceutical and food industries implies the expansion of the range of medicinal types, taking into account the experience of traditional and Tibetan medicine. The search for new medicinal and food plants requires a comprehensive study of flora and vegetation, comprehensive ecological and biological

\footnotetext{
* Corresponding author: doky2015@yandex.ru
} 
research on the basis of generalization, clarification and addition of theoretical, methodological and practical recommendations.

Population studies can be considered one of the priority areas of ecological and biological research. Identification of the population confinement of species to existing ecological and coenotic conditions, determination of demographic parameters, study of the development dynamics, methods of self-maintenance and survival strategies serve as the basis for the rational use of plant resources.

The work of many authors is devoted to the study of medicinal plants used in scientific and folk medicine. Resource science, ontogenetic and cenopopulation, introduction studies, identification of thickets of industrial importance, recommendations on scientifically grounded volumes of raw materials procurement, ecological-biological, biochemical and pharmacognostic studies, study of the technology of drying and processing of plant materials while preserving the maximum amount of biologically active substances, study the impact of recreational activities on the components of biocomplexes and other aspects considered by the authors will help to solve the problem of biodiversity conservation [1 24].

When developing the problems of preserving the biological diversity of wild-growing medicinal plants, the question inevitably arises about the criteria for an approach to the procurement of raw materials for valuable plant species. The dynamic structure of cenopopulations of medicinal plants, as a rule, is not taken into account when collecting and preparing raw materials. In this connection, the aim of our research was to study the dynamic structure of wild medicinal plant cenopopulations in the conditions of Cisbaikalia.

\section{Materials and Methods}

Study of cenopopulations of 16 species of medicinal plants (Achillea asiatica Serg., Artemisia frigida Willd., Chamaenerion angustifolium (L.) Holub, Comarum palustre L., Geranium pratense L., Dasiphora fruticosa (L.) Rydb., Fragaria orientalis Losinsk., Mentha arvensis L., Pulmonaria mollis Wulfen ex Hornem., Potentilla anserina L., Potentilla bifurca L., Ribes spicatum E. Robson, Rosa majalis Herrm., Rubus matsumuranus Levl. et Vaniot, Spiraea salicifolia L., Vaccinium uliginosum L.) conducted by us on the territory of Cisbaikalia (Alar, Bokhansky, Ziminsky, Irkutsk, Nizhneudinsky, Olkhonsky regions) from 1999-2012.

The determination of the age composition and number of cenopopulations of medicinal plants was carried out in accordance with the method of T.A. Rabotnov, taking into account the methodology for studying the age structure of the populations that make up the community of V.D. Aleksandrova, criteria for identifying age states and features of the course of ontogenesis in plants of various biomorphs of L.I. Vorontsova, L.E. Gatsuk, V.N. Egorova and others, L.B. Zaugolnova, L.A. Zhukova, A.S. Komar, O.V. Smirnova, as well as the principles and methods of studying the age structure of cenopopulations by Yu.A. Zlobina, E.L. Nukhimovsky [25].

When assessing each age state, three groups of features were taken into account (according to the classification of life forms of Rabotnov-Uranov): a) morphostructure of individuals and its change in ontogenesis; b) the state of integration of elements; c) the sequence of passing the stages of morphogenesis. The age state was determined mainly by the aboveground parts: the number, shape and size of leaves, the number and length of shoots, the presence of flowers and fruits. Sometimes, to clarify the age state, they dug up individual shoots or dug up the whole plant [25].

Individuals belonging to the same age state were combined into one age group. The selection of age groups of individuals was carried out in accordance with the classification of age states of T.A. Rabotnov, indexes of age states were proposed by A.A. Uranov [25]. 
When assessing the dynamic structure of cenopopulations, we relied on the theory of A.A. Uranova, L.A. Zhukova, including with co-authors, according to which the dynamic processes occurring in populations reflect the age coefficients, recovery index, development rate [25]. The age index was determined by the formula:

$$
\Delta=\Sigma \mathrm{ki} \mathrm{mi} / \Sigma \mathrm{ki}, \text { where }
$$

$\mathrm{ki}$ is the size of each age group,

$\mathrm{mi}$ is the "weight" of the age of one individual in each age group:

$$
\mathrm{mi}=1 / 1+\mathrm{e}^{-\mathrm{x}}
$$

The development rate of cenopopulations was determined by the formula proposed by L.A. Zhukova:

$$
\mathrm{U} \Delta=\Delta 2-\Delta 1 / \mathrm{t} 2-\mathrm{t} 1, \text { where }
$$

$\Delta 1$ is initial age, $\Delta 2$ is final age, $\mathrm{t} 2-\mathrm{t} 1$ is time interval.

The recovery index $\left(\mathrm{J}_{\mathrm{R}}, \%\right)$ was calculated to characterize the dynamics of selfmaintenance:

$$
\mathrm{J}_{\mathrm{B}}=\Sigma \mathrm{j} \rightarrow \mathrm{v} \quad / \Sigma \mathrm{g} 1 \rightarrow \mathrm{g} 3 \times 100 \%
$$

\section{Results}

Cisbaikalia is a region of the south of Eastern Siberia, located in the center of the Asian continent. The territory of Cisbaikalia, according to the botanical-geographical zoning, belongs to the Eurasian coniferous-forest region, the Euro-Siberian subregion and is characterized by a plain or plateau relief, the predominance of pine forests. Dark coniferous forests with the participation of spruce, cedar, fir and larch are located in elevated areas. Birch and aspen forests, as well as mixed forests with the participation of small-leaved species, are common at the site of fires.

The results of the ontogenetic structure studies of cenopopulations of the studied medicinal plants are shown in Table 1.

According to theory of A.A. Uranova and L.A. Zhukova, the dynamic processes taking place in populations reflect the age coefficients, recovery index and development rate (Table 2).

The plant communities of medicinal plants in all regions were identical, and therefore, the

\begin{tabular}{|c|c|c|c|c|c|c|c|c|c|c|c|c|c|}
\hline \multirow{2}{*}{ View } & \multirow{2}{*}{$\begin{array}{c}\text { Research } \\
\text { area }\end{array}$} & \multirow{2}{*}{$\begin{array}{c}\text { Plant } \\
\text { Communities }\end{array}$} & \multirow{2}{*}{ Year } & \multicolumn{10}{|c|}{ Ontogenetic structure, $\%$} \\
\hline & & & & $\mathrm{p}$ & $\mathrm{j}_{1}$ & $\mathrm{j}_{2}$ & im & $\mathrm{v}$ & $\mathrm{g}_{1}$ & $\mathrm{~g}_{2}$ & $g_{3}$ & ss & $\mathrm{s}$ \\
\hline \multirow{8}{*}{$\begin{array}{l}\text { Achillea } \\
\text { asiatica }\end{array}$} & \multirow{2}{*}{ Alar } & \multirow{8}{*}{$\begin{array}{l}\text { Pine-birch } \\
\text { forest, forb, } \\
\text { forb meadow }\end{array}$} & 2005 & 0.1 & 0.2 & 1.4 & 3.8 & 12.5 & 29.0 & 38.3 & 13.6 & 1.1 & - \\
\hline & & & 2011 & 0.3 & 0.3 & 0.6 & 1.5 & 13.0 & 20.1 & 37.6 & 24.3 & 2.3 & - \\
\hline & \multirow{2}{*}{ Irkutsk } & & 2005 & 0.8 & 1.0 & 1.2 & 1.5 & 16.0 & 32.5 & 30.1 & 15.9 & 1.0 & - \\
\hline & & & 2010 & 0.4 & 0.2 & 0.5 & 1.1 & 27.0 & 28.9 & 23.4 & 17.5 & 1.0 & - \\
\hline & \multirow{2}{*}{ Ziminsky } & & 2005 & 0.3 & 0.9 & 1.1 & 1.9 & 10.0 & 50.6 & 30.2 & 4.7 & 0.3 & \\
\hline & & & 2011 & 0.3 & 1.4 & 0.5 & 1.7 & 7.6 & 67.3 & 18.4 & 1.3 & 1.5 & - \\
\hline & \multirow{2}{*}{$\begin{array}{l}\text { Nizhneudi } \\
\text { nsky }\end{array}$} & & 2005 & 0.6 & 0.8 & 1.5 & 2.0 & 17.1 & 45.5 & 20.6 & 10.3 & 1.6 & - \\
\hline & & & 2011 & 0.1 & 0.5 & 1.0 & 1.3 & 26.2 & 39.6 & 22.7 & 6.2 & 2.4 & - \\
\hline \multirow{2}{*}{$\begin{array}{l}\text { Artemisia } \\
\text { frigida }\end{array}$} & \multirow{2}{*}{ Olkhonsky } & Grass-forb real & 2000 & 1.2 & 1.6 & 1.6 & 2.0 & 2.3 & 51.2 & 25.2 & 10.2 & 4.7 & - \\
\hline & & steppes & 2011 & 0.2 & 0.3 & 0.4 & 0.7 & 0.8 & 29.5 & 29.7 & 28.9 & 9.5 & - \\
\hline Chamaener & Irkutsk & Bereznyak, & 2000 & 0.6 & 2.0 & 6.5 & 10.3 & 13.2 & 18.4 & 40.0 & \begin{tabular}{|l}
7.0 \\
\end{tabular} & - & 2.0 \\
\hline
\end{tabular}
experimental data on the studied cenopopulations of 16 species were summarized by us and presented for the studied regions of the study.

Table 1. Dynamics of raw material stocks and age structure of medicinal plant cenopopulations. 


\begin{tabular}{|c|c|c|c|c|c|c|c|c|c|c|c|c|c|}
\hline \multirow{4}{*}{$\begin{array}{c}\text { View } \\
\text { ion } \\
\text { angustifoliu } \\
m\end{array}$} & \multirow{4}{*}{$\begin{array}{l}\text { Research } \\
\text { area }\end{array}$} & \multirow{4}{*}{\begin{tabular}{|c|} 
Plant \\
Communities \\
mixed fireweed \\
forest, grass- \\
fireweed \\
meadow
\end{tabular}} & \multirow{2}{*}{ Year } & \multicolumn{10}{|c|}{ Ontogenetic structure, $\%$} \\
\hline & & & & $\mathrm{p}$ & $\mathrm{j}_{1}$ & $\mathrm{j}_{2}$ & $\mathrm{im}$ & $\mathrm{v}$ & $\mathrm{g}_{1}$ & $\mathrm{~g}_{2}$ & $\mathrm{~g}_{3}$ & ss & $\mathrm{s}$ \\
\hline & & & 2006 & 1.0 & 4.6 & 8.8 & 9.2 & 9.7 & 26.9 & 33.8 & 5.0 & - & 1.0 \\
\hline & & & 2011 & 0.9 & 3.8 & 8.5 & 8.7 & 10.4 & 20.0 & 42.4 & 4.2 & - & 1.1 \\
\hline \multirow[b]{2}{*}{$\begin{array}{l}\text { Comarum } \\
\text { palustre }\end{array}$} & \multirow[b]{2}{*}{ Irkutsk } & \multirow{2}{*}{$\begin{array}{c}\text { Bereznyak } \\
\text { rotational, } \\
\text { horsetail; } \\
\text { soddy sedge } \\
\text { meadow } \\
\end{array}$} & 2006 & 0.6 & 0.6 & 0.8 & 4.4 & 11.0 & 23.2 & 30.6 & 25.9 & 2.3 & 0.6 \\
\hline & & & 2012 & 0.6 & 0.6 & 0.6 & 2.3 & 3.3 & 9.7 & 42.5 & 35.4 & 4.0 & 1.0 \\
\hline \multirow{7}{*}{$\begin{array}{c}\text { Dasiphora } \\
\text { fruticosa }\end{array}$} & \multirow{2}{*}{ Alar } & \multirow{7}{*}{ Yernik forb } & 2005 & 1.4 & 1.6 & 2.0 & 3.2 & 8.0 & 46.1 & 35.8 & 1.3 & 0.6 & - \\
\hline & & & 2012 & 2.1 & 2.4 & 3.8 & 5.6 & 6.3 & 41.1 & 37.6 & 0.7 & 0.4 & - \\
\hline & \multirow{3}{*}{ Irkutsk } & & 1999 & 0.1 & 0.5 & 0.3 & 3.7 & 15.0 & 40.2 & 36.9 & 2.4 & 0.9 & - \\
\hline & & & 2005 & 0.2 & 0.7 & 2.3 & 5.0 & 12.4 & 39.8 & 35.5 & 2.5 & 1.6 & - \\
\hline & & & 2011 & 0.3 & 0.3 & 1.1 & 3.6 & 10.7 & 38.2 & 40.4 & 3.6 & 1.8 & - \\
\hline & \multirow{2}{*}{ Olkhonsky } & & 2006 & 0.1 & 0.2 & 1.6 & 2.8 & 5.2 & 40.6 & 39.3 & 7.7 & 2.5 & - \\
\hline & & & 2011 & 0.6 & 1.3 & 1.3 & 2.2 & 2.2 & 31.8 & 47.8 & 9.6 & 3.2 & - \\
\hline \multirow{7}{*}{$\begin{array}{l}\text { Fragaria } \\
\text { orientalis }\end{array}$} & \multirow{3}{*}{ Alar } & \multirow{7}{*}{$\begin{array}{c}\text { Grass- } \\
\text { strawberry-forb } \\
\text { meadow }\end{array}$} & 2000 & 1.0 & 1.8 & 2.5 & 3.8 & 6.4 & 29.5 & 48.6 & 3.7 & 2.0 & 0.7 \\
\hline & & & 2005 & 1.3 & 1.5 & 1.7 & 1.5 & 10.2 & 32.0 & 40.4 & 6.5 & 3.7 & 1.2 \\
\hline & & & 2012 & 0.8 & 1.0 & 1.2 & 1.4 & 1.8 & 34.7 & 58.0 & 0.8 & 0.2 & 0.1 \\
\hline & 7 iminsky & & 2005 & 0.6 & 1.3 & 2.0 & 2.5 & 4.5 & 22.2 & 45.5 & 10.9 & 10.3 & 0.2 \\
\hline & Ziminsky & & 2011 & 0.1 & 0.5 & 0.6 & 0.9 & 1.1 & 19.2 & 43.0 & 18.6 & 15.0 & 1.0 \\
\hline & & & 2005 & 0.5 & 0.8 & 1.4 & 2.1 & 8.2 & 53.3 & 27.7 & 5.0 & 1.0 & - \\
\hline & & & 2010 & 0.3 & 0.6 & 1.0 & 1.3 & 3.7 & 52.4 & 29.5 & 9.5 & 1.7 & - \\
\hline \multirow{8}{*}{$\begin{array}{l}\text { Geranium } \\
\text { pratense }\end{array}$} & \multirow{3}{*}{ Alar } & \multirow{8}{*}{$\begin{array}{l}\text { Grain- } \\
\text { geranium-forb } \\
\text { meadow }\end{array}$} & 2000 & 1.5 & 4.2 & 5.0 & 1.33 & 27.9 & 40.1 & 16.9 & 2.4 & 0.6 & - \\
\hline & & & 2005 & 1.0 & 2.8 & 3.5 & 16.5 & 20.4 & 31.0 & 20.0 & 4.0 & 0.8 & - \\
\hline & & & 2012 & 4.4 & 2.6 & 4.4 & 14.8 & 16.5 & 27.4 & 19.1 & 9.0 & 1.7 & 0.1 \\
\hline & 7 iminsky & & 2005 & 0.6 & 0.9 & 8.3 & 0.4 & 25.2 & 30.7 & 30.2 & 3.4 & \begin{tabular}{|l|}
0.3 \\
\end{tabular} & - \\
\hline & Ziminsky & & 2011 & 1.3 & 2.0 & 15.6 & 3.7 & 17.3 & 36.5 & 16.3 & 6.5 & 0.8 & - \\
\hline & & & 2000 & 1.7 & 3.4 & 6.7 & 9.9 & 14.4 & 38.8 & 22.0 & 1.6 & 1.5 & - \\
\hline & Irkutsk & & 2005 & 3.0 & 4.6 & 6.3 & 1.5 & 9.3 & 38.1 & 30.2 & 5.8 & 1.2 & - \\
\hline & & & 2010 & 6.2 & 9.3 & 3.1 & 5.2 & 17.0 & 33.4 & 22.5 & 2.9 & \begin{tabular}{|l|}
0.3 \\
\end{tabular} & 0.1 \\
\hline & & & 2005 & 0.3 & 1.5 & 1.4 & 1.7 & 7.6 & 56.3 & 26.0 & 3.5 & 1.7 & - \\
\hline & & & 2012 & 1.4 & 2.3 & 2.7 & 3.6 & 4.1 & 52.2 & 23.9 & 5.8 & 4.0 & - \\
\hline Mentha & Irkout & Yernik forb. & 2006 & 0.8 & 0.9 & 3.1 & 8.8 & 30.3 & 45.6 & 8.6 & 1.4 & 0.5 & - \\
\hline arvensis & Irkutsk & $\begin{array}{l}\text { sedge-horseta1 } \\
\text { meadow }\end{array}$ & 2010 & 0.4 & 1.6 & 4.5 & 1.2 & 5.8 & 47.4 & 38.5 & 2.8 & 0.8 & - \\
\hline & Olkh & & 2006 & 0.1 & 0.1 & 0.6 & 1.7 & 9.3 & 58.4 & 27.9 & 1.6 & 0.3 & - \\
\hline & & & 2011 & 1.0 & 2.0 & 4.0 & 4.0 & 6.7 & 50.5 & 26.0 & 4.8 & 1.0 & - \\
\hline Potentilla & kutsk & Grass-goose- & 2001 & 0.02 & 1.2 & 1.2 & 4.88 & 29.4 & 24.4 & 24.4 & 12.2 & 2.3 & - \\
\hline & Irkutsk & leafed me & 2010 & 0.02 & 0.05 & 0.06 & 0.85 & 10.69 & 44.35 & 24.3 & 16.52 & 3.16 & - \\
\hline & & Forb-cold & 2006 & 0.1 & \begin{tabular}{|l|}
0.1 \\
\end{tabular} & 0.3 & 4.7 & 8.0 & 39.0 & 43.0 & 4.0 & 0.8 & - \\
\hline $\begin{array}{l}\text { Potentilla } \\
\text { bifurca }\end{array}$ & Olkhonsky & $\begin{array}{l}\text { wormwood } \\
\text { mountain } \\
\text { steppes }\end{array}$ & 2011 & 0.2 & 0.5 & 1.6 & 2.9 & 5.6 & 35.2 & 46.2 & 6.5 & 1.3 & - \\
\hline & & & 2005 & 0.3 & 1.9 & 2.8 & 11.6 & 17.5 & 38.0 & 23.9 & \begin{tabular}{|l|l}
9.0 \\
\end{tabular} & - & 1.0 \\
\hline & & & 2012 & 0.4 & 4.0 & 5.0 & 9.9 & 21.5 & 30.0 & 22.0 & 5.5 & - & 1.7 \\
\hline & $7 \mathrm{im}$ & & 2005 & 0.6 & 2.0 & 1.8 & 3.8 & 29.2 & 22.6 & 22.4 & 16.2 & - & 1.4 \\
\hline $\begin{array}{l}\text { Pulmonarta } \\
\text { mollis }\end{array}$ & Ziminsky & Bereznyak forb & 2011 & 0.2 & 1.1 & 1.6 & 2.5 & 28.7 & 26.0 & 19.7 & 718.0 & - & 2.2 \\
\hline & & & 1999 & 0.5 & 2.7 & 3.2 & 16.9 & 14.2 & 37.2 & 23.1 & 2.0 & - & 0.2 \\
\hline & Irkutsk & & 2005 & 0.8 & 3.0 & 6.6 & 13.5 & 15.2 & 33.8 & 25.2 & \begin{tabular}{|l|l}
2 & 1.7 \\
\end{tabular} & - & 0.2 \\
\hline & & & 2010 & 0.9 & 5.1 & 8.3 & 14.1 & 8.9 & 38.4 & 21.5 & \begin{tabular}{|l|l|}
52.3 \\
\end{tabular} & - & 0.5 \\
\hline Ribes & & Yonile & 2005 & 0.3 & 3.5 & 7.2 & 10.2 & 10.4 & 27.8 & 33.0 & 7.0 & 0.6 & - \\
\hline spicatum & Alar & Yernik forb & 2012 & 0.6 & 1.3 & 4.6 & 5.3 & 6.0 & 19.9 & 56.3 & \begin{tabular}{|l|l}
35.3 \\
\end{tabular} & \begin{tabular}{|l|}
0.7 \\
\end{tabular} & - \\
\hline
\end{tabular}




\begin{tabular}{|c|c|c|c|c|c|c|c|c|c|c|c|c|c|}
\hline \multirow{4}{*}{ View } & \multirow{2}{*}{$\begin{array}{l}\text { Research } \\
\text { area }\end{array}$} & \multirow{4}{*}{$\begin{array}{c}\text { Plant } \\
\text { Communities }\end{array}$} & \multirow{2}{*}{ Year } & \multicolumn{10}{|c|}{ Ontogenetic structure, $\%$} \\
\hline & & & & $\mathrm{p}$ & $\mathrm{j}_{1}$ & $\mathrm{j}_{2}$ & $\mathrm{im}$ & $\mathrm{V}$ & $\mathrm{g}_{1}$ & $\mathrm{~g}_{2}$ & $\mathrm{~g}_{3}$ & ss & $\mathrm{s}$ \\
\hline & \multirow{2}{*}{ Irkutsk } & & 2005 & 0.1 & 4.0 & 5.5 & 10.1 & 10.6 & 24.7 & 41.0 & 3.8 & 0.2 & - \\
\hline & & & 2012 & 0.1 & 2.8 & 4.6 & 7.4 & 10.7 & 25.5 & 46.5 & 1.4 & 0.5 & 0.5 \\
\hline \multirow{12}{*}{$\begin{array}{c}\text { Rosa } \\
\text { majalis }\end{array}$} & \multirow{3}{*}{ Alar } & \multirow{12}{*}{$\begin{array}{c}\text { Bereznyak } \\
\text { cereal. sedge }\end{array}$} & 2001 & 0.7 & 1.2 & 3.5 & 9.0 & 26.3 & 22.1 & 18.2 & 18.0 & 1.0 & - \\
\hline & & & 2005 & 0.2 & 0.8 & 0.7 & 10.6 & 15.9 & 30.3 & 25.6 & 13.6 & 2.0 & 0.3 \\
\hline & & & 2012 & 0.4 & 0.1 & 0.5 & 3.0 & 8.3 & 21.9 & 39.4 & 21.2 & 4.5 & 0.7 \\
\hline & \multirow{3}{*}{ Ziminsky } & & 2001 & 0.1 & 0.1 & 2.4 & 4.6 & 17.0 & 18.3 & 20.3 & 19.5 & 9.8 & 7.9 \\
\hline & & & 2005 & 0.3 & 0.9 & 1.5 & 1.5 & 6.6 & 20.0 & 19.5 & 29.7 & 9.6 & 10.4 \\
\hline & & & 2011 & 0.1 & 0.5 & 0.6 & 2.0 & 3.0 & 8.0 & 22.2 & 40.4 & 10.1 & 13.1 \\
\hline & \multirow{3}{*}{ Irkutsk } & & 2001 & 0.7 & 2.6 & 5.4 & 10.9 & 21.9 & 33.7 & 21.5 & 2.6 & 0.7 & - \\
\hline & & & 2005 & 0.2 & 1.8 & 5.3 & 5.0 & 21.4 & 26.0 & 38.5 & 1.3 & 0.5 & - \\
\hline & & & 2012 & 0.5 & 3.9 & 6.1 & 7.7 & 16.6 & 19.4 & 43.7 & 1.6 & 0.5 & - \\
\hline & \multirow{3}{*}{$\begin{array}{c}\text { Nizhneudin } \\
\text { sky }\end{array}$} & & 2000 & 0.3 & 0.3 & 1.5 & 15.4 & 18.0 & 32.5 & 24.3 & 4.8 & 2.0 & 0.9 \\
\hline & & & 2005 & 0.4 & 1.7 & 3.6 & 12.5 & 10.1 & 21.6 & 37.6 & \begin{tabular}{|l|}
7.2 \\
\end{tabular} & 3.9 & 1.4 \\
\hline & & & 2012 & 0.2 & 2.5 & 5.0 & 7.5 & 8.3 & 19.2 & 42.5 & 9.1 & 4.1 & 1.6 \\
\hline \multirow{4}{*}{$\begin{array}{c}\text { Rubus } \\
\text { matsumura } \\
\text { nus }\end{array}$} & \multirow{2}{*}{ Alar } & \multirow{4}{*}{$\begin{array}{l}\text { Mixed grass- } \\
\text { forb forest }\end{array}$} & 2001 & 2.6 & 2.9 & 6.4 & 10.6 & 18.2 & 20.0 & 24.3 & 10.8 & 3.7 & 0.5 \\
\hline & & & 2010 & 4.3 & 6.3 & 6.7 & 7.5 & 13.5 & 17.5 & 19.0 & 14.0 & 9.1 & 2.1 \\
\hline & \multirow{2}{*}{ Irkutsk } & & 2001 & 1.8 & 1.5 & 2.4 & 6.0 & 18.0 & 22.2 & 41.5 & 4.2 & 2.0 & 0.4 \\
\hline & & & 2010 & 2.6 & 3.5 & 3.8 & 5.5 & 20.6 & 25.6 & 32.0 & 3.2 & 2.6 & 0.6 \\
\hline \multirow{4}{*}{$\begin{array}{c}\text { Spiraea } \\
\text { salicifolia }\end{array}$} & \multirow{2}{*}{ Alar } & \multirow{4}{*}{ Yernik forb } & 2006 & 0.6 & 0.8 & 2.8 & 9.6 & 23.3 & 39.3 & 19.5 & 4.0 & 0.1 & - \\
\hline & & & 2011 & 0.3 & 0.4 & 3.3 & 8.4 & 19.1 & 37.6 & 24.1 & \begin{tabular}{|l|}
6.7 \\
\end{tabular} & 0.1 & - \\
\hline & \multirow{2}{*}{ Irkutsk } & & 2006 & 0.9 & 0.7 & 1.5 & 7.0 & 12.0 & 43.5 & 30.6 & 2.1 & 1.7 & - \\
\hline & & & 2011 & 1.4 & 1.8 & 4.7 & 9.7 & 10.4 & 32.4 & 34.4 & 2.9 & 2.2 & 0.1 \\
\hline \multirow{6}{*}{$\begin{array}{l}\text { Vaccinium } \\
\text { uliginosum }\end{array}$} & \multirow{3}{*}{ Irkutsk } & \multirow{6}{*}{$\begin{array}{c}\text { Blueberry pine } \\
\text { forest }\end{array}$} & 1999 & 0.3 & 0.4 & 1.6 & 2.3 & 10.0 & 22.0 & 56.5 & 3.9 & 2.2 & 0.8 \\
\hline & & & 2005 & 1.2 & 1.5 & 2.0 & 3.9 & 13.7 & 36.5 & 37.3 & 2.5 & 1.0 & 0.4 \\
\hline & & & 2011 & 1.5 & 2.6 & 4.2 & 6.3 & 6.5 & 20.7 & 52.7 & 4.0 & 1.0 & 0.5 \\
\hline & \multirow{3}{*}{ Olkhonsky } & & 2000 & 0.1 & 0.4 & 0.9 & 5.3 & 14.4 & 30.1 & 40.6 & 6.4 & 1.8 & - \\
\hline & & & 2005 & 0.1 & 0.2 & 1.6 & 3.9 & 8.4 & 34.0 & 35.3 & 11.6 & 4.7 & 0.2 \\
\hline & & & 2010 & 0.7 & 0.8 & 1.7 & 3.4 & 10.1 & 28.5 & 35.9 & 13.5 & 5.2 & 0.2 \\
\hline
\end{tabular}

Note: $p$ - seedlings (shoots), j (j1, j2) - juvenile, im - immature, v - virginal (young vegetative, adult vegetative), g1 - young generative, g2 - middle-aged generative, g3 - old generative, ss - subsenile (old vegetative), s - senile.

Table 2. Dynamics of medicinal plant cenopopulations.

\begin{tabular}{|c|c|c|c|c|c|c|c|}
\hline \multirow[b]{2}{*}{ Research area } & \multicolumn{7}{|c|}{ Coefficients of cenopopulation dynamic } \\
\hline & \multicolumn{3}{|c|}{ Age of cenopopulation, $\Delta$} & \multicolumn{3}{|c|}{$\begin{array}{c}\text { Recovery index, } \\
\mathrm{J}_{\mathrm{R}}\end{array}$} & $\begin{array}{l}\text { Development } \\
\quad \text { rate, } U\end{array}$ \\
\hline \multicolumn{8}{|c|}{ Achillea asiatica } \\
\hline Research year & 2000 & 2005 & $\begin{array}{l}2010 \\
2011 .\end{array}$ & 2000 & 2005 & $\begin{array}{l}2010- \\
2011 \\
\end{array}$ & $2000-2011$ \\
\hline Alar & - & 0.396 & 0.456 & - & 22.1 & 18.8 & 1.00 \\
\hline Ziminsky & - & 0.335 & 0.314 & - & 16.1 & 12.8 & -0.35 \\
\hline Irkutsk & - & 0.385 & 0.375 & - & 25.6 & 41.4 & -0.22 \\
\hline Nizhneudinsky & - & 0.337 & 0.319 & - & 28.5 & 40.4 & -0.30 \\
\hline \multicolumn{8}{|c|}{ Artemisia frigida } \\
\hline Research year & 2000 & 2005 & 2011 & 2000 & 2005 & 2011 & $2000-2011$ \\
\hline Olkhonsky & 0.385 & - & 0.526 & 8.6 & - & 2.4 & 1.28 \\
\hline \multicolumn{8}{|c|}{ Chamaenerion angustifolium } \\
\hline Research year & 2000 & 2006 & 2011 & 2000 & 2006 & 2011 & $2000-2011$ \\
\hline Irkutsk & 0.343 & 0.434 & 0.399 & 49.6 & 50.5 & 48.5 & 0.50 \\
\hline \multicolumn{8}{|c|}{ Comarum palustre } \\
\hline Research year & 2000 & 2006 & 2012 & 2000 & 2006 & 2012 & $2000-2012$ \\
\hline
\end{tabular}




\begin{tabular}{|c|c|c|c|c|c|c|c|}
\hline \multirow{3}{*}{$\begin{array}{c}\text { Research area } \\
\text { Irkutsk }\end{array}$} & \multicolumn{7}{|c|}{ Coefficients of cenopopulation dynamic } \\
\hline & \multicolumn{3}{|c|}{ Age of cenopopulation, $\Delta$} & \multicolumn{3}{|c|}{$\begin{array}{c}\text { Recovery index, } \\
\mathrm{J}_{\mathrm{R}}\end{array}$} & \multirow{2}{*}{$\begin{array}{c}\begin{array}{c}\text { Developmen } \\
\text { rate, } \mathrm{U}\end{array} \\
1.33 \\
\end{array}$} \\
\hline & - & 0.446 & 0.366 & - & 20.8 & 9.8 & \\
\hline \multicolumn{8}{|c|}{ Dasiphora fruticosa } \\
\hline Research year & 1999 & $\begin{array}{r}2005- \\
2006\end{array}$ & $\begin{array}{r}2011- \\
2012 \\
\end{array}$ & 1999 & $\begin{array}{c}2005- \\
2006\end{array}$ & $\begin{array}{r}2011- \\
2012 \\
\end{array}$ & $1999-2012$ \\
\hline Alar & - & 0.328 & 0.346 & - & 19.8 & 25.4 & 0.25 \\
\hline Irkutsk & 0.128 & 0.335 & 0.361 & 24.7 & 26.7 & 19.5 & 1.94 \\
\hline Olkhonsky & - & 0.551 & 0.426 & - & 6.5 & 8.5 & -2.48 \\
\hline \multicolumn{8}{|c|}{ Fragaria orientalis } \\
\hline Research year & 2000 & 2005 & $\begin{array}{r}2010- \\
2012 \\
\end{array}$ & 2000 & 2005 & $\begin{array}{r}2010- \\
2012 \\
\end{array}$ & $2000-2012$ \\
\hline Alar & 0.384 & 0.394 & 0.396 & 17.9 & 18.9 & 5.8 & 0.10 \\
\hline Ziminsky & - & 0.467 & 0.547 & - & 13.3 & 3.8 & 1.33 \\
\hline Irkutsk & - & 0.339 & 0.379 & - & 14.5 & 7.2 & 0.78 \\
\hline Nizhneudinsky & - & 0.348 & 0.418 & - & 15.2 & 11.4 & 1.17 \\
\hline \multicolumn{8}{|c|}{ Geranium pratense } \\
\hline Research year & 2000 & 2005 & $\begin{array}{c}2010- \\
2012\end{array}$ & 2000 & 2005 & $\begin{array}{c}2010- \\
2012\end{array}$ & $2000-2012$ \\
\hline Alar & 0.252 & 0.253 & 0.326 & 64.4 & 78.5 & 68.9 & 0.61 \\
\hline Ziminsky & - & 0.294 & 0.259 & - & 53.9 & 65.3 & -0.56 \\
\hline Irkutsk & 0.264 & 0.316 & 0.253 & 55.2 & 29.7 & 58.6 & -0.11 \\
\hline \multicolumn{8}{|c|}{ Mentha arvensis } \\
\hline Research year & 2000 & $\begin{array}{c}2005- \\
2006\end{array}$ & $\begin{array}{c}2010- \\
2012\end{array}$ & 2000 & $\begin{array}{c}2005- \\
2006\end{array}$ & $\begin{array}{c}2010- \\
2012\end{array}$ & $2005-2012$ \\
\hline Alar & - & 0.333 & 0.346 & - & 14.0 & 15.4 & 0.19 \\
\hline Irkutsk & - & 0.258 & 0.353 & - & 24.8 & 11.4 & 2.36 \\
\hline Olkhonsky & - & 0.324 & 0.319 & - & 13.7 & 20.0 & -0.09 \\
\hline \multicolumn{8}{|c|}{ Potentilla bifurca } \\
\hline Research year & 1999 & 2006. & 2011 & 1999 & 2006 & 2011 & $2006-2011$ \\
\hline Irkutsk & - & 0.368 & 0.395 & - & 15.0 & 11.9 & 0.52 \\
\hline \multicolumn{8}{|c|}{ Potentilla anserina } \\
\hline Research year & 2001 & $\begin{array}{r}2005- \\
2006 \\
\end{array}$ & 2010 & 2001 & $\begin{array}{r}2005- \\
2006\end{array}$ & 2010 & $2001-2010$ \\
\hline Irkutsk & 0.387 & - & 0.403 & 60.0 & - & 13.7 & 0.17 \\
\hline \multicolumn{8}{|c|}{ Pulmonaria mollis } \\
\hline Research year & 1999 & 2005 & $\begin{array}{l}2010 \\
2012 \\
\end{array}$ & 1999 & 2005 & $\begin{array}{l}2010- \\
2012\end{array}$ & $1999-2012$ \\
\hline Alar & - & 0.281 & 0.280 & - & 43.0 & 71.2 & -0.005 \\
\hline Ziminsky & - & 0.328 & 0.359 & - & 61.3 & 53.5 & 0.50 \\
\hline Irkutsk & 0.258 & 0.257 & 0.252 & 58.2 & 64.4 & 60.0 & -0.02 \\
\hline \multicolumn{8}{|c|}{ Ribes spicatum } \\
\hline Research year & 2001 & 2005 & 2012 & 2001 & 2005 & 2012 & $2001-2012$ \\
\hline Alar & - & 0.316 & 0.390 & - & 46.4 & 21.1 & 1.05 \\
\hline Irkutsk & - & 0.320 & 0.338 & - & 43.4 & 34.8 & 0.25 \\
\hline \multicolumn{8}{|c|}{ Rosa majalis } \\
\hline Research year & $\begin{array}{l}2000- \\
2001\end{array}$ & 2005 & $\begin{array}{c}2011- \\
2012 \\
\end{array}$ & $\begin{array}{l}2000- \\
2001\end{array}$ & 2005 & $\begin{array}{l}2011- \\
2012\end{array}$ & $2000-2012$ \\
\hline Alar & 0.329 & 0.355 & 0.470 & 68.4 & 40.5 & 14.5 & 1.27 \\
\hline Ziminsky & 0.481 & 0.564 & 0.650 & 41.6 & 27.2 & 8.7 & 1.69 \\
\hline Irkutsk & 0.256 & 0.305 & 0.322 & 73.9 & 51.0 & 52.9 & 0.59 \\
\hline Nizhneudinsky & 0.300 & 0.365 & 0.399 & 57.2 & 42.0 & 32.9 & 0.82 \\
\hline \multicolumn{8}{|c|}{ Rubus matsumuranus } \\
\hline
\end{tabular}




\begin{tabular}{|c|c|c|c|c|c|c|c|}
\hline \multirow{3}{*}{$\begin{array}{l}\text { Research area } \\
\text { Research year }\end{array}$} & \multicolumn{7}{|c|}{ Coefficients of cenopopulation dynamic } \\
\hline & \multicolumn{3}{|c|}{ Age of cenopopulation, $\Delta$} & \multicolumn{3}{|c|}{$\begin{array}{c}\text { Recovery index, } \\
\mathrm{J}_{\mathrm{R}}\end{array}$} & \multirow{2}{*}{$\begin{array}{c}\begin{array}{c}\text { Development } \\
\text { rate, U }\end{array} \\
2001-2010 \\
\end{array}$} \\
\hline & 2001 & 2005 & 2010 & 2001 & 2005 & 2010 & \\
\hline Alar & 0.322 & - & 0.410 & 68.7 & - & 67.1 & 0.98 \\
\hline Irkutsk & 0.345 & - & 0.309 & 41.0 & - & 55.0 & -0.38 \\
\hline \multicolumn{8}{|c|}{ Spiraea salicifolia } \\
\hline Research year & 2000 & 2006 & 2011 & 2000 & 2006 & 2011 & $2006-2011$ \\
\hline Alar & - & 0.267 & 0.300 & - & 58.2 & 45.7 & 0.65 \\
\hline Irkutsk & - & 0.318 & 0.319 & - & 27.8 & 38.1 & 0.005 \\
\hline \multicolumn{8}{|c|}{ Vaccinium uliginosum } \\
\hline Research year & $\begin{array}{l}1999- \\
2000\end{array}$ & $\begin{array}{c}2005- \\
2006\end{array}$ & $\begin{array}{c}2010- \\
2011\end{array}$ & $\begin{array}{l}1999- \\
2000\end{array}$ & $\begin{array}{c}2005- \\
2006\end{array}$ & $\begin{array}{l}2010- \\
2011\end{array}$ & $1999-2011$ \\
\hline Irkutsk & 0.411 & 0.334 & 0.375 & 17.3 & 27.8 & 25.3 & -0.30 \\
\hline Olkhonsky & 0.366 & 0.408 & 0.417 & 27.3 & 17.4 & 20.5 & 0.50 \\
\hline
\end{tabular}

\section{Discussion}

The results of the analysis of the cenopopulation dynamics of medicinal plants for the studied period from 1999 to 2012, showed that the cenopopulation of Achillea asiatica in the Alar region is normal, long-lived, but slowly developing, with a tendency towards aging $(\mathrm{U}=1)$. Gradual aging is also observed in the coenopopulation of Rubus matsumuranus (U $=0.98)$ of the Alar region, Fragaria orientalis, Rosa majalis and Spiraea salicifolia $(\mathrm{U}=$ 0.005-0.65) $(\mathrm{U}=0.59-1.69)$ of the Alar, Ziminsky, Irkutsk and Nizhneudinsky regions $(\mathrm{U}=$ $0.1-1.33)$; Artemisia frigida $(\mathrm{U}=1.28)$ of the Olkhonsky region; Comarum palustre $(\mathrm{U}=$ $1.33)$, Mentha arvensis $(\mathrm{U}=2.36)$, Potentilla bifurca $(\mathrm{U}=0.52)$, Potentilla anserina $(\mathrm{U}=$ $0.17)$ of the Irkutsk region; Pulmonaria mollis $(\mathrm{U}=0.50)$ of the Ziminsky region; Ribes spicatum $(\mathrm{U}=0.25-1.05)$, Rosa acicularis $(\mathrm{U}=0.30-1.29)$ of the Alar and Irkutsk regions; Vaccinium uliginosum $(\mathrm{U}=0.50)$ of the Olkhonsky region.

The cenopopulation of Chamaenerion angustifolium is stable and rapidly developing $\left(\mathrm{J}_{\mathrm{R}}\right.$ $=48.5-50.5)$, the species is regenerated both by seed and vegetative means. A high recovery index was also noted in Pulmonaria mollis $\left(\mathrm{J}_{\mathrm{R}}=43-71.2\right)$. Fluctuations in the dynamics of age and recovery index for Geranium pratense $\left(\mathrm{J}_{\mathrm{R}}=29.7-78.5\right)$, Potentilla anserina $\left(\mathrm{J}_{\mathrm{R}}=13.7-60\right)$, Pulmonaria mollis $\left(\mathrm{J}_{\mathrm{R}}=43-71.2\right)$, Rosa majalis $\left(\mathrm{J}_{\mathrm{R}}=8.7-73.9\right)$, Rubus matsumuranus $\left(\mathrm{J}_{\mathrm{R}}=41-68.7\right)$, Spiraea salicifolia $\left(\mathrm{J}_{\mathrm{R}}=27.8-58.2\right)$ and other species over the studied period of time, can be associated with biomorphological features of the species, for example, with the ability of the rhizomes of Chamaenerion angustifolium and other species to partially die off, fall out of the clone structure and quickly replace the fallen shoots with young vegetative particles, as well as with climatic features of the year (dry growing season), anthropogenic (irrational procurements of raw materials, pasture digression) and pyrogenic factors.

The cenopopulation of Dasiphora fruticosa is quite stable, periodically renewing by seed. The moderately pronounced seed renewal of Dasiphora fruticosa $\left(\mathrm{J}_{\mathrm{R}}=6.5-26.7\right)$ is possibly associated with the weak ability of seeds to germinate in a dry climatic period. Development towards rejuvenation is observed in the Olkhonsky cenopopulation of Dasiphora fruticosa $(\mathrm{U}=-2.48)$, a fairly young Alar cenopopulation $(\mathrm{U}=0.25)$.

Gradual rejuvenation is also observed in the cenopopulation of Achillea asiatica of the Ziminsky, Irkutsk and Nizhneudinsky regions $(\mathrm{U}=-0.22-0.35)$, Geranium pratense of the Ziminsky and Irkutsk regions $(\mathrm{U}=-0.11-0.56)$, Rubus matsumuranus $(\mathrm{U}=-0.38)$ of the Irkutsk region. The Irkutsk coenopopulations of Vaccinium uliginosum $(\mathrm{U}=-0.30)$ and others stable and dynamically developing coenopopulations of Pulmonaria mollis are 
developed with rejuvenation, while development towards rejuvenation, with good seed renewal, is observed in coenopopulations of Pulmonaria mollis of the Alar $(\mathrm{U}=-0.005)$ and Irkutsk regions $(\mathrm{U}=-0.02)$.

\section{Conclusions}

The stable and dynamically developing cenopopulations of medicinal plants, with a high recovery index $\left(J_{R} \geq 50\right)$, with a tendency to develop towards rejuvenation, include the cenopopulations of Geranium pratense of the Ziminsky region; gradual rejuvenation is observed in the coenopopulations of Pulmonaria mollis of the Alar and Irkutsk regions, Rubus matsumuranus of the Irkutsk region. Irkutsk cenopopulations of Chamaenerion angustifolium, Rosa majalis, Ziminsky cenopopulations of Pulmonaria mollis, Alar cenopopulations of Geranium pratense, Rubus matsumuranus, Spiraea salicifolia develop with a tendency towards aging.

The results of the analysis of the cenopopulation dynamics of the studied species for the studied period of time (1999-2012) made it possible to identify stable, dynamically developing cenopopulations and recommend them for use. Determining the demographic parameters of useful plants, studying the dynamics of development, methods of selfmaintenance and survival strategies of species serve as the basis for the rational use of plant resources.

\section{References}

1. A.Y. Astashenkov, V.A. Cheryomushkina, A.B. Myrzagaliyeva, B.Z. Medeubayeva, Ontogenesis, estimation of coenopopulation state and component composition in Nepeta densiflora Kar. Et Kir. (Lamiaceae) individuals of East Kazakhstan. International, Journal of Environmental Studies, 76(4), 634-647 (2019). doi: 10.1080/00207233.2018.1509496.

2. I.L. Bukharina, K.E. Vedernikov, A.S. Pashkova Morphophysiologic traits of spruce trees in conditions of Izhevsk, Contemporary Problems of Ecology, 9(7), 853-862. (2016).

3. E.A. Efremov, I.D. Zykova, A.A Efremov Component composition of essential oil and some extractives Pulmonaria mollis Hornem, Siberian Medical Journal, 122 (7), 125128 (2013).

4. M.M. Ishmuratova, A.R. Ishbirdin, M.M. Cherosov, N.I. Baryshnikova, E.N. Suleimanova Ecological-phytocenotic, population, resource characteristics of Valeriana alternifolia Ledeb. Bulletin of the Ammosov North-Eastern Federal University, 6 (62), 17-24 (2017).

5. T.V. Krasnova, M.A. Safonov, Z.N. Ryabinina Bioresource potential of the Central Orenburg region, Publishing house: Publishing and printing complex "University", (2014).

6. E. G. Khudonogova, M. A. Rachenko, N. G. Dubrovsky, O. A. Popova, T. T. Taisayev, Stocks of raw materials of wild medicinal plants in the Western Baikal Region, In: IOP Conference Series: Earth and Environmental Science, 315 (2019). https://doi.org/10.1088/1755-1315/315/7/072002.

7. E. Khudonogova, S. Tretyakova, A. Mikhlyaeva Ecological features of useful plants in natural populations of the Western Baikal Region. In: International Multidisciplinary Scientific GeoConference Surveying Geology and Mining Ecology Management, SGEM, 19, 301-306 (2019). https://doi.org/10.5593/sgem2019/5.2/S20.037. 
8. E. Khudonogova, O. Zatsepina, S. Polovinkina, M. Rachenko, Seed germination of woody and shrubby introduced species. IOP Conference Series: Earth and Environmental Science, 316 (2019). https://doi.org/10.1088/1755-1315/316/1/012021.

9. E.G. Khudonogova, A.A. Mikhlyaeva, S.V. Polovinkina, Ontogenetic development of Hedysarum alpinum L. in Prebaikalia. Global Journal of Science Frontier Research, 20(3), 35-40, (2020)

10. E.B. Kolegova, V.A. Cheryomushkina, Ontogenetic structure and assessment of state of Thymus mongolicus (Lamiaceae) coenopopulations in Southern Siberia, Contemporary Problems of Ecology, 8 (2), 155-161 (2015). DOI: 10.1134/S1995425515020079.

11. A.V. Kuritsyn, V.D. Belonogova, G.I. Oleshko, Resources of medicinal plant materials in the southern regions of the Perm Territory, 3, 11-13 (2007).

12. B.B. Namzalov, S.V. Zhigzhitzhapova, N.G. Dubrovsky Wormwoods of Buryatia: diversity analysis, ecological-geographical features, and chemotaxonomy of section abrotanum, Acta Biologica Sibirica, 5(3), 178-187 (2019). doi: 10.14258/abs.v5.i3.6589.

13. N.A. Nikolaeva, I.A. Parygin, S.V. Tretyakova, Ecological characteristics of useful plants in the Baikal region, Topical issues of agricultural science, 21, 27-34 (2016).

14. Yu.A. Plotnikova, E.S. Barysheva, I.V. Mikhailova, The effect of anthropogenic load on the pharmacological activity of medicinal herbs growing on the territory of the Orenburg region (review), Problems of ecology of the Southern Urals. Collection of materials of the IX All-Russian scientific-practical conference with international participation. 229-233 (2019).

15. T.V. Polozova, T.P. Antsupova, Natural resource potential of wild plants in the Republic of Buryatia. In the collection: State and development of production and processing of livestock products, hunting and fishing, Materials of the international scientific and practical conference dedicated to the 80th anniversary of the Faculty of Technology, 153-156 (2018).

16. E.A. Ponomarenko, O.V. Ryabinina, The impact of recreational activities on the components of biocomplexes in the Olkhon region and Olkhon island, Bulletin of $\operatorname{IrGSKhA,~78,~13-18~(2017).~}$

17. G.O. Osmanova, S.V. Kozyreva, O.P. Vedernikova, The role of ontogenetic herbarium in studying intrapopulation biodiversity, Pakistan Journal of Botany, 48(4), 1495-1500 (2016).

18. J.S. Sattarov, S.Kh. Vyshegurov, R.R Galeev Monitoring the species diversity of medicinal plants typical for the south slope of Hissar Ridge, Agronomy Research. 18(2), 543-553 (2018).

19. E.B. Talovskaya, Ontogenetic structure of coenopopulations of Thymus baicalensis (Lamiaceae) in the south of Siberia. Bulletin of Tomsk State University, 1 (33), 39-49 (2016).

20. S.A. Kholboeva, B.B. Namzalov, E.V. Bukharova, O.V. Imetkhenova,: On the diversity of shrub communities in the vegetation of the Selenga midlands (Western Transbaikalia), The flora of Asian Russia, 1 (17), 55-63 (2015).

21. I.A. Khudonogov, Resource-saving principles of IR-power supply control in the processes of processing medicinal plants into health-improving tea. Bulletin of KrasGAU, 1 (28), 127-131 (2009).

22. I.A. Khudonogov, E.G. Khudonogova, Classification and energy technology system for processing wild and cultivated raw materials by infrared heating. In the collection: 
Joint activities of agricultural producers and scientific organizations in the development of the agro-industrial complex of Central Asia. Collection of materials of the international scientific and practical conference: 4 parts. Ministry of Agriculture of the Russian Federation, Department of Science and Technology Policy and Education, 112-116 (2008).

23. E.G. Khudonogova, Medicinal plants of Cisbaikalia for tea and health drinks, Bulletin of the Russian Academy of Agricultural Sciences. 1, 87-89 (2009).

24. E.G. Khudonogova, N.A. Nikolaeva, N.Yu. Chernigovskaya, Raw materials of wild medicinal plants in Cisbaikalia, Topical issues of agricultural science, 3, 13-21 (2012).

25. E.G. Khudonogova, Biology, ecology and productivity of useful plants in Cisbaikalia: analysis, forecasting and optimization of use: abstract of doctors of biological sciences thesis (2015). 